\title{
Abstract: Technique for studying chemisorption on substrates with complex band structures ${ }^{\text {a) }}$
}

\author{
S. L. Cunningham and W. H. Weinberg ${ }^{\text {b) }}$ \\ Division of Chemistry and Chemical Engineering, California Institute of Technology, Pasadena, California 91125
}

V. M. Tapilin

Institute of Catalysis, Novosibirsk, 630090 USSR

(Received 19 September 1977; accepted 22 November 1977)

PACS numbers: 68.45.Da

Chemisorption calculations of single adatoms on infinitely periodic surfaces using the Green's function approach have been limited heretofore to simple model systems due to the difficulty in obtaining the appropriate Green's functions. ${ }^{1}$ In this paper a new technique is presented which has a number of numerical advantages making it possible to obtain the necessary Green's functions for materials with realistic and complex band structures.

The problem is approached by determining the Green's functions for four subsystems. Whereas only the last of the four contains the desired information regarding the chemisorbed system, the method relies on the fact that the first Green's function is easy to calculate, and through a series of steps, the other three can be found sequentially. The method first determines $G^{\circ}$, the Green's function for a single twodimensional layer of atoms having the structure of a layer in the bulk crystal. Second, the Green's function $G$ for the bulk crystal is calculated; this is the difficult step. Third, the Green's function $G^{s}$ for a crystal with a surface is found. Fourth, the Green's function $G^{a}$ which contains the desired information regarding the chemisorbed system, is obtained.

For the first step, interactions between layers are ignored. The Schrödinger matrix equation in the LCAO formalism is

$$
\sum_{j} H_{i j}(\mathbf{k}) a_{j}=\epsilon(\mathbf{k}) a_{i},
$$

where $\mathbf{k}$ is the two-dimensional wave vector for the layer, and the Hamiltonian matrix is $(N \times N)$, where $N$ is the number of orbitals on each atom. Even for complex systems, this matrix is not very large. The Green's function can be calculated directly as

$$
G_{i j}^{o}=\sum_{\kappa} \frac{a_{i \kappa} a_{j \kappa}}{E-\epsilon_{\kappa}} \delta_{\ell \ell^{\prime}},
$$

where $E$ is the energy variable, $\kappa$ labels the eigenvalue and associated eigenvector, and the indices $\ell$ and $\ell^{\prime}$ refer to layer number. This matrix is infinite in dimension due to the $\ell$ and $\ell^{\prime}$ indices, but it is made up of identical $(N \times N)$ blocks along the diagonal. The first step, then, requires finding the eigenvalues and eigenvectors of the $(N \times N)$ matrix $H$.

For the second step, the Green's function for the bulk is obtained by writing a Dyson equation

$$
\begin{aligned}
& G_{i j}(O \ell)=G_{i j}^{o}(O \ell) \\
& \quad+\sum_{i^{\prime} j^{\prime} \ell^{\prime}} G_{i j^{\prime}}^{o}(O O) V_{i^{\prime} j^{\prime}}\left(O \ell^{\prime}\right) G_{j^{\prime} j}\left(\ell^{\prime} \ell\right),
\end{aligned}
$$

where the first layer index is set to zero and where the perturbation matrix $V$ is that part of the bulk crystal Hamiltonian that connects layers $O$ and $\ell^{\prime} \neq O$. Define $M$ to be the largest integer for which $V_{i j}(O M) \neq O$. Then Eq. (3) breaks into two types of equations depending upon the value of the index $\ell$.

For $\ell>M$, Eq. (3) becomes simply

$$
G_{i j}(O \ell)=\sum_{i^{\prime} j^{\prime}} \sum_{\ell^{\prime}=\ell-M}^{\ell+M} G_{i i^{\prime}}^{o}(O O) V_{i^{\prime} j^{\prime}}\left(\ell^{\prime} \ell\right) G_{j^{\prime} j}\left(\ell^{\prime} O\right) .
$$

This equation can be solved by defining a stepping operator $q$ such that

$$
G_{i j}(O \ell)=q^{\ell} G_{i j}(O O) .
$$

Upon substituting Eq. (5) into (4), we obtain an eigenvalue equation (for the case $M=1$ )

$$
\sum_{j^{\prime}}\left(A_{i j^{\prime}}-Q \delta_{i j^{\prime}}\right) G_{j^{\prime} j}(O O)=O
$$

where

$$
A_{i j^{\prime}}=\sum_{i^{\prime}} G_{i i^{\prime}}^{o}(O O) V_{i^{\prime} j^{\prime}}(O 1)
$$

and

$$
Q=\left(q+q^{-1}\right)^{-1} .
$$

There are $2 N$ solutions for $q$ from Eqs. (6)-(8). By choosing the $N$ solutions which satisfy $|q| \leqslant 1$, we have the set of homogeneous solutions to Eq. (3).

For the case of $\ell<M$ in Eq. (3) we obtain a more complex equation. The general solution is constructed from a linear combination of the homogeneous solutions with arbitrary coefficients. This gives the bulk Green's function as

$$
G_{i j}(O \ell)=\sum_{\alpha=1}^{N} b_{\alpha j} q_{\alpha}^{\ell} G_{i j}^{\alpha}(O O),
$$

where the values of $q_{\alpha}$ and $G_{i j}^{\alpha}(O O)$ are the $\alpha$ th eigenvalue and eigenvector from Eq. (6) and where the coefficients $b_{\alpha j}$ are determined from the set of linear equations 


$$
\sum_{\alpha=1}^{N} B_{i \alpha} b_{\alpha j}=G_{i j}^{o}(O O)
$$

where

$$
B_{i \alpha}=\sum_{j^{\prime}}\left(\delta_{i j^{\prime}}-2 q_{\alpha} A_{i j^{\prime}}\right) G_{j^{\prime} i}^{\alpha}(O O) .
$$

The second step, then, requires finding the eigenvalues and eigenvectors of the $(N \times N)$ matrix $A$ and solving $N$ times the $(N \times N)$ set of linear equations in Eq. (10). This is the difficult step. The bulk Green's function is constructed from Eq. (9).

For the third step, the Green's function for the surface is simply found by inverting the Dyson equation

$$
G_{i j}^{s}(O O)=G_{i j}(O O)-\sum_{i^{\prime} j^{\prime}} G_{i i^{\prime}}(O 1) V_{i^{\prime} j^{\prime}}(1 O) G_{j^{\prime} j}(O O),
$$

where the minus sign is due to the fact that the surface can be formed merely by subtracting all interactions across an imaginary plane. Hence, we obtain

$$
G_{i j}^{s}(O O)=\sum_{j^{\prime}}\left(D^{-1}\right)_{i j^{\prime}} G_{j^{\prime} j}(O O),
$$

where

$$
D_{i j}=\delta_{i j}+\sum_{i^{\prime}} G_{i i^{\prime}}(O 1) V_{i^{\prime} j}(O 1) .
$$

The third step, then, requires inverting an $(N \times N)$ matrix.

The fourth step of finding the Green's function for the chemisorbed system $G^{a}$ is standard ${ }^{1,2}$ and will not be repeated here.

All of the Green's functions mentioned depend implicitly on $\mathbf{k}$, the wave vector parallel to the surface. To obtain the total Green's function, a numerical sum over the special points in the surface Brillouin zone is performed. ${ }^{3}$

Previous methods for finding the surface Green's functions involve either solving a continued fraction 4,5 or performing an integration over the $z$ component of the wave vector. ${ }^{6}$ Both of these methods encounter numerical difficulties and become prohibitively time consuming when applied to systems with complex band structures and multiple neighbor interactions. The method presented here, while appearing to be more complex for model systems, does not have the same drawbacks as these previous methods when applied to realistic systems.

To further elucidate the procedure, we have applied the formalism to examine the chemisorption of a one-level adatom in the on-site configuration on the (100) surface of a two-band substrate with the $f c c$ structure. This problem has not been considered previously. It is shown that when a single level interacts with a multiple band substrate, increasing the interaction between the adatom and only one band significantly affects the electron density in the other bands.

a) Complete paper to appear in Physical Review. Supported by National Science Foundation Grant No. CHE77-10629.

b) Alfred P. Sloan Foundation Fellow, and Camille and Henry Dreyfus Foundation Teacher-Scholar.

${ }^{1}$ See, for example, T. B. Grimley, Prog. Surf. Membr. Sci. 9, 71 (1975).

${ }^{2}$ W. Ho, S. L. Cunningham, and W. H. Weinberg, Surf. Sci. 54, 139 (1976); Surf. Sci. 62, 662 (1977).

${ }^{3} \mathrm{~S}$. L. Cunningham, Phys. Rev. B 10, 4988 (1974).

${ }^{4}$ R. Haydock, V. Heine, and M. J. Kelly, J. Phys. C 5, 2845 (1972).

${ }^{5}$ F. Cyrot-Lackmann, M. C. Desjonqueres, and J. P. Gaspard, J. Phys. C 7, 925 (1974).

${ }^{6}$ W. Ho, S. L. Cunningham, W. H. Weinberg, and L. Dobrzynski, Phys. Rev. B 12, 3027 (1975). 